\title{
Identifying Optimal Nanofluid Synthesis Conditions for Applications in Solar Thermal Concentrators
}

\author{
Anurag Pramanik ${ }^{* 1}$, Harjit Singh ${ }^{1}$, S. Suresh ${ }^{2}$, Ram Chandra ${ }^{3}$, Virendra Kumar Vijay ${ }^{3}$ \\ ${ }^{1}$ Institute of Energy Futures, Brunel University London, Uxbridge UB8 3PH, UNITED KINGDOM \\ Email: Anurag.Pramanik@brunel.ac.uk
}

2 Department of Mechanical Engineering, National Institute of Technology, Tiruchirappalli 620015, Tamil Nadu, INDIA

${ }^{3}$ Centre for rural Development and Technology, Indian Institute of Technology Delhi, Hauz Khas, New Delhi 110016, INDIA

\begin{abstract}
For solar thermal applications, a novel idea is to employ directly radiation-absorbing working fluids to completely replace metallic receiver tubes. Advances in nanotechnology has enabled development of nanofluids, an optimized mix of nanoparticles (NPs) and a base fluid, which can absorb a large proportion of the solar spectrum. A nanofluid comprising of carbon NPs in ethylene glycol has been synthesized for solar thermal applications. The method of preparation and sonication conditions have been studied to evaluate their effect on nanofluid stability and its solar absorptivity. Nanofluid with a NP concentration of $40-300 \mathrm{mg} / \mathrm{l}$ was synthesized by adding a surfactant (Sodium dodecyl sulfate) and carbon NPs (<100 nm) in ethylene glycol followed by sonication for different durations (up to $60 \mathrm{~min}$ ). Transmittance of the nanofluid was measured using UV-vis spectrophotometer after preparation and at pre-determined time intervals to evaluate the effect of sonication duration. Solar absorptivity of nanofluid was found to increase with increasing NP concentration. Sonication time of 60 min was found to be sufficient to form a uniform suspension with low transmittance (and therefore, high absorptivity). Continuous stirring maintained the nanofluid optical performance for longer durations with negligible changes in solar absorptivity. The optimal conditions for synthesizing a stable nanofluid for longer durations have been identified and are described here.
\end{abstract}

KEYWORDS: Carbon, Nanofluid, Nanoparticles, Solar absorptivity, Solar thermal concentrators.

\section{INTRODUCTION}

Climate change is one of the biggest existential threat to our society. Greenhouse gas emissions are leading to an increase in global temperatures and in the long term, can induce catastrophic effects [1]. There is an immediate need to reduce the dependence on unsustainable resources such as coal and petroleum. Solar energy is a promising renewable and sustainable option with various applications including electricity generation, desalination, and thermal applications [2]. The total amount of solar energy on earth is very high but distributed over a large area and intermittent in nature. Hence, efficient solar radiation collection and storage are essential components for solar thermal applications. 
The two main components of the solar collection include a heat transfer fluid and solar thermal concentrator. The heat transfer fluid can be innovated to directly absorb the incident solar radiation to completely substitute for selectivity coated metallic receivers. Significant advances have been made in developing novel designs of solar thermal concentrators which focus the solar radiation with high concentration ratio [2]. These use ethylene glycol, Therminol VP-1 (special formulation of diphenyl oxide and biphenyl) and, molten salts, etc. as working fluids passing through a selectively coated metallic receiver. However, they lack the absorption capacity for the solar radiation and therefore can't be used directly for solar thermal applications [3]. Addition of NPs $(1-100 \mathrm{~nm})$ to these fluids to form a uniform suspension, also called 'nanofluid', can significantly enhance (by upto 7 orders of magnitude) the fluids' solar energy absorption capability and therefore, the use of nanofluids holds potential in solar thermal applications [3, 4]. Optimizing the nanofluid properties and stability so that it absorbs solar radiation across a broad spectrum of wavelength, especially in the visible and near infrared range (300-2500 nm), is an essential step to develop a new class of directly absorbing solar collectors $[5,6]$. Developing robust protocols for nanofluid synthesis which will ensure long-term stability of such nanofluids is, therefore, a key step for its wider application in thermal applications [7-11].

In this study, we have used carbon NPs $(<100 \mathrm{~nm})$ and ethylene glycol based nanofluid which show excellent absorption spectra in the visible and near infrared range [4]. The main objective of this study is to identify optimal conditions for maintaining stability of nanofluid over a prolonged duration of use. We have studied the effects of NP concentration and duration of sonication on nanofluid properties. Our results highlight the importance of these parameters when synthesizing carbon NPs based nanofluids for solar thermal applications.

\section{MATERIAL AND METHODS}

\subsection{Synthesis of nanofluid}

Carbon NPs (Sigma-Aldrich) were weighed (40-300 mg/l) and added to ethylene glycol (Sigma-Aldrich) the base fluid. A non-ionic surfactant, $0.1 \%$ (wt/vol) sodium dodecyl sulphate (Sigma-Aldrich) was also added to the mixture. The mixture was suspended using a bath-type sonicator for different durations (30 and 60 minutes).

\subsection{UV-Vis characterization}

The nanofluid was characterized at various time points after synthesis using a UV-Vis spectrophotometer. The samples were enclosed in a Suprasil quartz cuvette with a path length of $1 \mathrm{~cm}$. Measurements were taken over the 320-1000 nm range. Transmittance spectra of the pure base fluid (ethylene glycol) was also measured for reference.

\section{RESULTS AND DISCUSSIONS}

The methodology for synthesis of nanofluid involves two step process [6]. First, the NPs are prepared as dry powders and in the second step, they are dispersed in the base fluid with aid of ultrasonic agitation. This is the most widely used and economical method for nanofluid synthesis. To further increase the stability of the nanofluid suspension, surfactant is added to improve the dispersibility of NPs [6]. Despite its simplicity, there are various parameters which can affect agglomeration of NPs. This can lead to precipitation of the NPs and result in reduction in absorbance of the nanofluid as well as clogging of pipes, etc. Hence, to identify the important aspects of nanofluid synthesis and storage, we varied several parameters and measured nanofluid stability using spectral absorbance analysis. An outline of the overall experimental strategy and different variables is shown in Figure 1. 


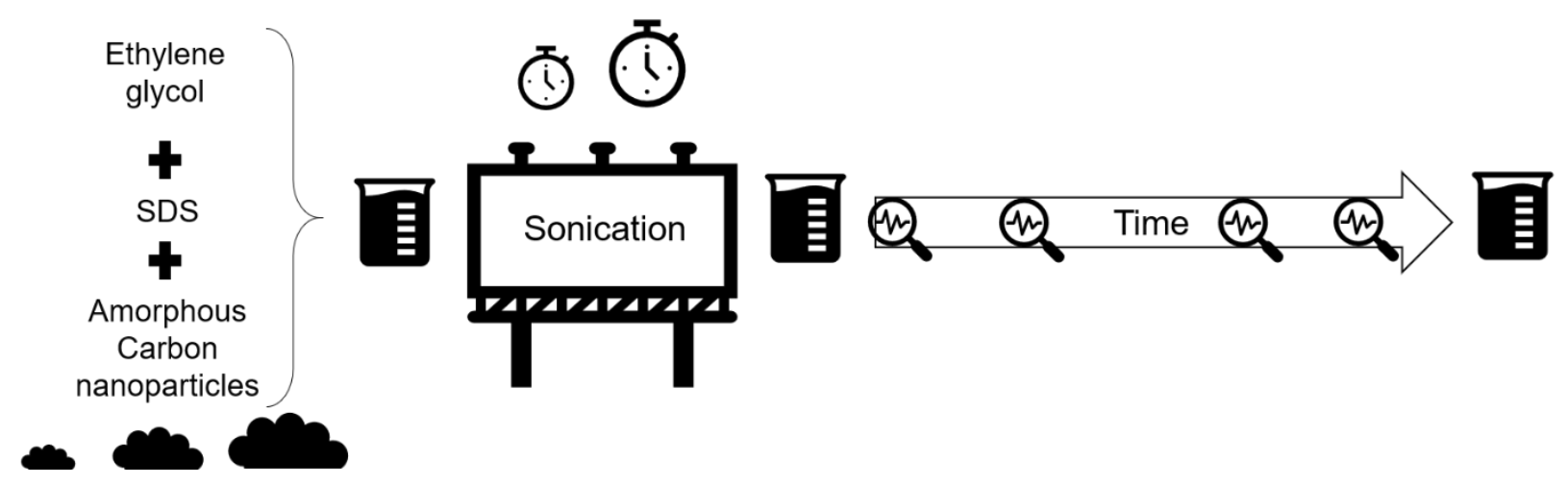

Figure 1: Outline of experiments used to assess nanofluid stability for long-term storage

Ethylene glycol is commonly used as a base fluid for solar thermal applications. The transmittance spectra for ethylene glycol is shown in Figure 2.

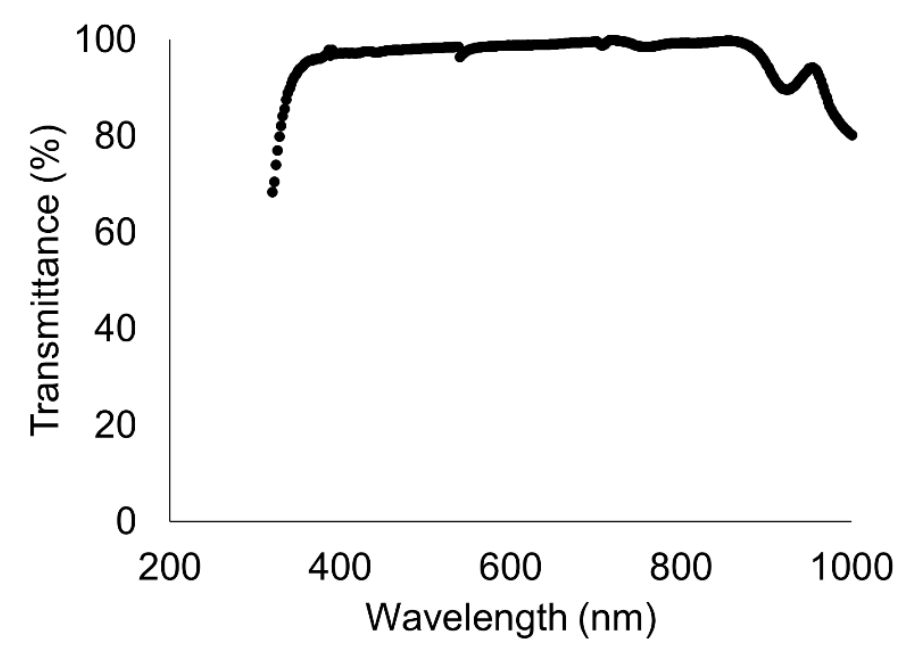

Figure 2: Spectral analysis of pure ethylene glycol

The range of wavelength chosen covers the visible and near infrared region (NIR) which accounts for more than $80 \%$ of the solar energy. It is observed that ethylene glycol has very high transmittance, hence very low absorbance.

\subsection{Effect of nanoparticle concentration on nanofluid spectral transmittance}

We next studied the effects of NP concentration on the absorption properties of nanofluid. We observed a concentration dependent exponential decline in transmittance (and hence, an increase in absorbance) of nanofluid (Figure 3b). It is important to note that increasing the concentration of NP beyond $100 \mathrm{mg} / \mathrm{L}$ does not improve the absorption by a significant factor. Hence, we chose $100 \mathrm{mg} / \mathrm{l}$ concentration for rest of the experiments in this study. 



Figure 3: Spectral properties of nanofluids synthesized. a) Spectral transmittance measured over a spectral wavelength of $320 \mathrm{~nm}$ to $1000 \mathrm{~nm}$. b) Average transmittance measured over the spectral region against concentration.

\subsection{Effect of sonication duration on spectral properties of nanofluid}

The effect of sonication duration on nanofluid stability was studied. It was observed that 60 minutes of sonication led to a lower transmittance suggesting that increasing the sonication duration leads to more efficient dispersion of NPs (Figure 4).

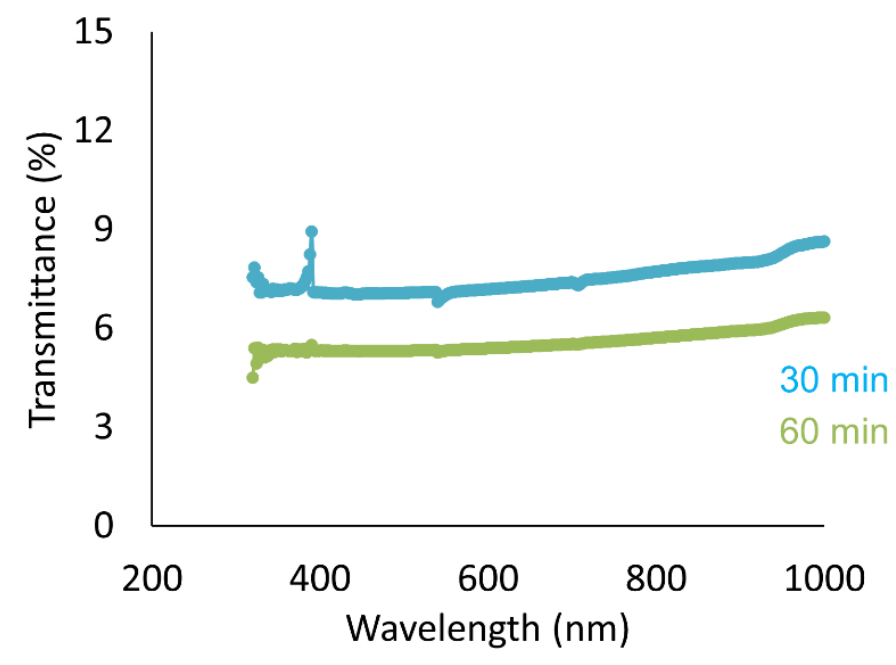

Figure 4: Sonication duration improves nanofluid properties. Spectral transmittance of nanofluid after sonication for 30 minutes and 60 minutes.

\subsection{Long term stability of nanofluid with continuous stirring}

We next studied the long-term effects of storage of nanofluid on nanofluid properties with continuous stirring. We observed negligible effects due to storage even after 7 days (Figure $5)$. 


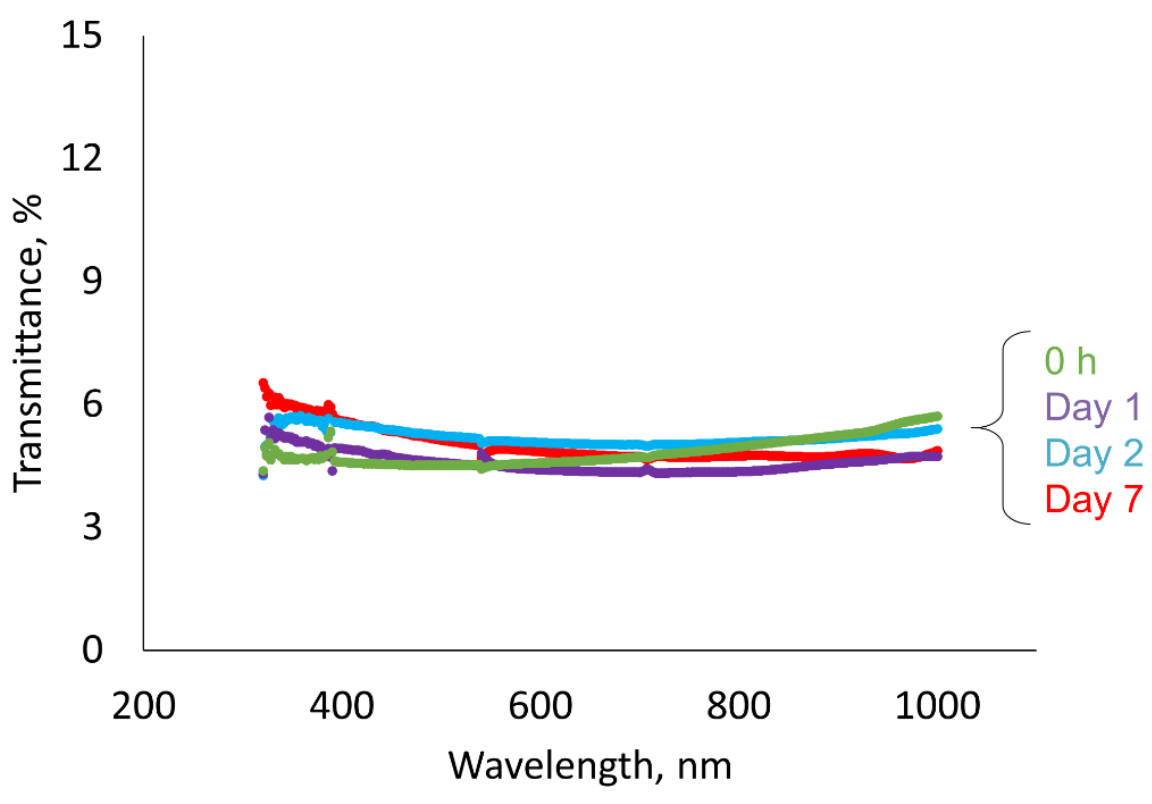

Figure 5: Nanofluid storage for longer duration. Spectral transmittance curves for nanofluid stored for different duration is shown.

\section{CONCLUSION}

This study has described the synthesis of carbon NPs- ethylene glycol nanofluids and measured the role of various synthesis conditions on nanofluids' optical performance parameters An exponential relationship between nanoparticle concentration and average transmittance was observed, which can be used to design nanofluid with desired spectral properties. A longer sonication duration was found to improve nanofluid properties (low transmittance). Finally, continuous stirring has been shown to aid in maintaining long term stability of nanofluids. The optimal conditions identified in this study are being employed to synthesize carbon NPs based nanofluids for solar thermal applications. Future studies will aim at evaluating the effectiveness of the developed nanofluids as working fluids in solar thermal collectors delivering heat at up to $300{ }^{\circ} \mathrm{C}$.

\section{ACKNOWLEDGMENTS}

Authors will like to acknowledge the funding received for this work from UK-India Education and Research Initiative (UKIERI) and Department of Science and Technology (DST) India through the grant agreement IND/CONT/GA/18-19/16.

\section{REFERENCES}

1. Nejat P, Jomehzadeh F, Taheri MM, Gohari M, Muhd MZ (2015) A global review of energy consumption, $\mathrm{CO} 2$ emissions and policy in the residential sector (with an overview of the top ten $\mathrm{CO} 2$ emitting countries). Renewable Sustainable Energy Reviews 43:843-862.

2. Tian Y, Zhao CY (2013) A review of solar collectors and thermal energy storage in solar thermal applications. Applied Energy 104:538-553 .

3. Khullar V, Tyagi H, Hordy N, Otanicar TP, Hewakuruppu Y, Modi P, Taylor RA (2014) Harvesting solar thermal energy through nanofluid-based volumetric 
absorption systems. International Journal of Heat and Mass Transfer 77:377-384 .

4. Hordy N, Rabilloud D, Meunier JL, Coulombe S (2014) High temperature and longterm stability of carbon nanotube nanofluids for direct absorption solar thermal collectors. Solar Energy 105:82-90 .

5. Rose BAJ, Singh H, Verma N, Tassou S, Suresh S, Anantharaman N, Mariotti D, Maguire $P$ (2017) Investigations into nanofluids as direct solar radiation collectors. Solar Energy 147:426-431 .

6. Yu W, Xie H (2012) A review on nanofluids: Preparation, stability mechanisms, and applications. Journal of Nanomaters 2012 .

7. Mahian O, Kianifar A, Kalogirou SA, Pop I, Wongwises S (2013) A review of the applications of nanofluids in solar energy. International Journal of Heat and Mass Transfer 57:582-594 .

8. Shah SNA, Shahabuddin S, Sabri MFM, Salleh MFM, Ali MA, Hayat N, Sidik NAC, Samykano M, Saidur R (2020) Experimental investigation on stability, thermal conductivity and rheological properties of rGO/ethylene glycol based nanofluids. International Journal of Heat and Mass Transfer 150:118981 .

9. Yu W, France DM, Routbort JL, Choi SUS (2008) Review and Comparison of Nanofluid Thermal Conductivity and Heat Transfer Enhancements. Heat Transfer Engineering 29:432-460 .

10. Mesgari S, Taylor RA, Hjerrild NE, Crisostomo F, Li Q, Scott J (2016) An investigation of thermal stability of carbon nanofluids for solar thermal applications. Solar Energy Materials and Solar Cells 157:652-659 .

11. Taylor RA, Phelan PE, Otanicar TP, Adrian R, Prasher R (2011) Nanofluid optical property characterization: towards efficient direct absorption solar collectors. Nanoscale Research Letters 6:225 . 